\title{
Preventive role of Tomato bushy stunt virus RNA-interference suppressor protein in plant immune response
}

Dildabek A.*, Akbassova A., Stamgaliyeva Z., Ilyasova B., Tleukulova Zh., Amanbayeva U., Zhangazin S., Iksat N., Masalimov Zh., Omarov R.

L.N. Gumilyov Eurasian National University, Nur-Sultan, Kazakhstan

*e-mail: dildabekaruzhan15@gmail.com

Complete resistance in plant to virus infection is referred to as immunity. Except protein defense factors, plants operate with RNA-interference defense system, which was firstly demonstrated for plant viruses. RNA-interference is a posttranscriptional RNA silencing process that is evolutionary conserved across eukaryotic kingdoms, which involved in development, differentiation, response to stress factors through regulation the levels of specific RNAs. As a countermeasure, viruses encode suppressor proteins, which disturb RNA-interference pathway. One of the best biochemically characterized suppressor is Tomato bushy stunt virus encoded P19 protein. During TBSV replication, abundantly accumulated single-stranded genomic RNAs and double-stranded RNAs performed as substrates for Dicer-mediated cleavage into duplex short interfering RNAs (siRNA). AGO-family proteins with siRNAs form RNA-induced silencing complex. This forms the catalytic entity for RNA degradation that uses the incorporated siRNA to specifically targeting complementary RNAs. The main function of P19 as suppressor protein is prevention the unwinding of viral siRNA and thus preventing the incorporation of singlestranded RNA into RISC complex, therefore - programming. The X-ray crystallographic structure of the P19 suppressor protein and specific siRNA complex revealed that caliper tryptophan residues on P19 dimers precisely measure the binding of 21-nucleotide siRNAs by P19 dimers. These siRNA duplexes have 2-nt 3' overhangs. P19 binds the duplex region of siRNAs. Usually, most suppressor proteins are multifunctional. P19 protein also appeared like pathogenicity factor. Inoculation of susceptible plant with TBSV P19-null mutant (TBSV $\triangle$ P19) leads to recovery. Cross-inoculation experiments with wild type TBSV, which was preliminary inoculated TBSV $\triangle \mathrm{P} 19$ leads to recovery phenotypes instead systemic collapse. 